\title{
Treadmill Exercise Improves Motor Function by Suppressing Purkinje Cell Loss in Parkinson Disease Rats
}

\author{
Jae-Min Lee', Tae-Woon Kim¹, Sang-Seo Park', Jin-Hee Han², Mal-Soon Shin, Baek-Vin Lim, Sang-Hoon Kim ${ }^{1,5}$, \\ Seung-Soo Baek ${ }^{5}$, Young Sam $\mathrm{Cho}^{6}$, Khae Hawn Kim \\ ${ }^{1}$ Department of Physiology, College of Medicine, Kyung Hee University, Seoul, Korea \\ ${ }^{2}$ Department of Anesthesiology and Pain Medicine, Kyung Hee Medical Center, College of Medicine, Kyung Hee University, Seoul, Korea \\ ${ }^{3}$ School of Global Sport Studies, Korea University, Sejong, Korea \\ ${ }^{4}$ Division of Leisure \& Sports Science, Department of Exercise Prescription, Dongseo University, Busan, Korea \\ ${ }^{5}$ Department of Sport \& Health Science, College of Natural Science, Sangmyung University, Seoul, Korea \\ ${ }^{6}$ Department of Urology, Kangbuk Samsung Hospital, Sungkyunkwan University School of Medicine, Seoul, Korea \\ ${ }^{7}$ Department of Urology, Gil Medical Center, Gachon University School of Medicine, Incheon, Korea
}

Purpose: Rotenone is the most widely used neurotoxin for the making Parkinson disease (PD) animal model. The neurodegenerative disorder PD shows symptoms, such as slowness of movements, tremor at resting, rigidity, disturbance of gait, and instability of posture. We investigated whether treadmill running improves motor ability using rotenone-caused PD rats. The effect of treadmill running on PD was also assessed in relation with apoptosis of cerebellar Purkinje cells.

Methods: Treadmill running was applied to the rats in the exercise groups for 30 minutes once a day for 4 weeks, starting 4 weeks after birth. We used rota-rod test for the determination of motor coordination and balance. In this experiment, terminal deoxynucleotidyl transferase-mediated dUTP nick end labeling (TUNEL) staining, immunohistochemistry for calbindin, glial fibrillary acidic protein (GFAP), Iba-1, and western blot analysis for Bax and Bcl-2 were performed.

Results: Treadmill running enhanced motor balance and coordination by preventing the loss of Purkinje cells in the cerebellar vermis. Treadmill running suppressed PD-induced expression of GFAP-positive reactive astrocytes and Iba-1-positive microglia, showing that treadmill running suppressed reactive astrogliosis and microglia activation. Treadmill running suppressed TUNEL-positive cell number and Bax expression and enhanced Bcl-2 expression, demonstrating that treadmill running inhibited the progress of apoptosis in the cerebellum of rotenone-induced PD rats.

Conclusions: Treadmill running improved motor ability of the rotenone-induced PD rats by inhibiting apoptosis in the cerebellum. Apoptosis suppressing effect of treadmill running on rotenone-induced PD was achieved via suppression of reactive astrocyte and inhibition of microglial activation.

Keywords: Parkinson disease; Rotenone, Cerebellum; Motor coordination; Treadmill exercise; Apoptosis

- Fund/Grant Support: This study was supported by a research grant from Astellas Pharma Korea Inc. (Seoul, Korea)

- Research Ethics: All animal experimental procedures were approved by the Institutional Animal Care and Use Committee of Kyung Hee University (KHUASP[SE]-16-151), and performed in accordance with the National Institute of Health Council for the management and use of laboratory animals.

- Conflict of Interest: KHK and YSC, associate editors of INJ, are the corresponding and 9th authors of this article. However, they played no role whatsoever in the editorial evaluation of this article or the decision to publish it. Except for that, no potential conflict of interest relevant to this article was reported.

Corresponding author: Khae Hawn Kim (iD http://orcid.org/0000-0002-7045-8004 Department of Urology, Gachon University School of Medicine, Gil Medical Center, 21 Namdong-daero 774 beon-gil, Namdong-gu, Incheon 21565, Korea E-mail: kimcho99@gilhospital.com / Tel: +82-32-460-3334 / Fax: +82-32-460-8414 Submitted: September 7, 2018 / Accepted after revision: October 12, 2018 (i) This is an Open Access article distributed under the terms of the Creative Commons Attribution Non-Commercial License (http://creativeenses/by-nc/4.0/) which permits unrestricted non-commercial use, distribution, and reproduction in any medium, provided the original work is properly cited. 


\footnotetext{
- HIGHLIGHTS

- Parkinson disease is a neurodegenerative disorder showing slowness of movements, tremor at resting, rigidity, disturbance of gait, and instability of posture.

- Treadmill running improved motor ability of the rotenone-induced Parkinson disease rats by inhibiting apoptosis in the cerebellum.

- Apoptosis suppressing effect of treadmill running on rotenone-induced Parkinson disease was achieved via suppression of reactive astrocyte and inhibition of microglial activation.
}

\section{INTRODUCTION}

The neurodegenerative disorder Parkinson disease (PD) shows symptoms, such as tremor, bradykinesia, rigidity, and postural instability (motor symptoms) as well as depression, apathy, sleep disorders, and erectile dysfunction (nonmotor symptoms) [1,2]. Neurotoxin-based models of PD elucidate the underlying mechanisms of the dopaminergic neuronal cell death [3]. Rotenone is a potent mitochondrial complex I inhibitor, and then it is the most widely used neurotoxin to make animal model of PD. Intravenous or subcutaneous injection of rotenone decreased the survival rate of rats, and in their surviving rats, immunoreactivity of the striatal tyrosine hydroxylase was decreased [4].

If the calcium-binding protein, calbindin D-28k (calbindin), in the Purkinje cells of cerebellum was deleted, different alterations in the cellular constituents and behaviors were occurred [5]. Long-term activation of cerebellar Purkinje cells was correlated with dopaminergic neuronal degeneration [6]. Inflammation is an important pathogenesis factor causing age-associated neurodegenerative disorders, including PD [7]. Purkinje cells can be detected by immunohistochemistry for calbindin, and decreased number of the calbindin-positive Purkinje cells was observed in the cerebellum of autistic rats [8].

Astrocyte-associated pathogenesis causes neurodegenerative diseases, for example, PD, Alzheimer disease, Huntington disease, and amyotrophic lateral sclerosis [9]. In response to PD or ischemia, reactive astrogliosis appears as a hallmark of these diseases [10-12]. Iba1 protein is an ionized calcium binding adaptor molecule 1 , and the expression of Iba1 was localized to macrophages and microglia [13]. Iba1 is a key molecule for ruffling of membrane and the phagocytosis of macrophages and microglia [14]. Microglia acts as a key initiator for rotenonecaused dopaminergic neuronal degeneration [15]. Activation of microglia has been suggested as the underlying mechanism of dopaminergic neuronal loss in the PD [16,17].

Apoptosis of neurons plays a crucial role for the PD induction $[18,19]$. Terminal deoxynucleotidyl transferase-mediated
dUTP nick end labeling (TUNEL) staining can detect DNA fragmentation, and apoptotic neuronal cell death is confirmed by this TUNEL staining [20-22]. Two molecules, known as Bax (pro-apoptotic molecule) and Bcl-2 (anti-apoptotic molecule), regulate the process of apoptotic cell death. During the apoptosis process, Bax is largely expressed and then promotes cell death, in contrast, Bcl-2 exerts a protective effect against cell death $[20,21]$.

Exercise is known to facilitate functional recovery from various brain injuries, including PD, Alzheimer disease, ischemia, and epilepsy $[17,20,21]$. In this study, we investigated whether treadmill running improves motor ability using rotenonecaused PD rats. In addition, the effect of treadmill running on PD was also assessed in relation with apoptosis of cerebellar Purkinje cells.

\section{MATERIALS AND METHODS}

\section{Animal Treatments}

We purchased Sprague-Dawley male rats $(80 \pm 10 \mathrm{~g}$, 4 weeks in age) for this experiment. All animal experimental procedures were approved by the Institutional Animal Care and Use Committee of Kyung Hee University (KHUASP[SE]-16-151), and performed in accordance with the National Institute of Health Council for the management and use of laboratory animals.

The rats were randomly classified into four groups ( $\mathrm{n}=10$ for each group): control, treadmill exercise, rotenone-injection, rotenone-injection and treadmill exercise group. Rotenone (Sigma Chemical Co., St. Louis, MO, USA) was diluted in the dimethyl sulfoxide:polyethylene glycol (1:1) solution. For the rats in the rotenone-injection groups, $3 \mathrm{mg} / \mathrm{kg}$ rotenone was injected, one time per a day during 14 days.

\section{Treadmill Running Protocol}

Treadmill running was applied to the rats in the exercise groups during 30 minutes, once time per a day for 4 weeks, starting 4 weeks after birth. The exercise speed was running at $2 \mathrm{~m} / \mathrm{min}$ 
during 5 minutes, at $3 \mathrm{~m} / \mathrm{min}$ during 5 minutes, and then at 5 $\mathrm{m} / \mathrm{min}$ for 20 minutes with no inclination.

\section{Rota-Rod Test}

To detect motor coordination and balance, rota-rod (Harvard Apparatus, Holliston, MA, USA) test was conducted, as the method described before $[23,24]$. Each rat was placed on the rotating rod (diameter, $4 \mathrm{~cm}$ ). Through the preliminary experiments, each rat was accustomed to the device at the speed of 4 rpm. After the preliminary experiments, the test trial was applied to the rats with increased speed from $4 \mathrm{rpm}$ to $20 \mathrm{rpm}$. The latency until falling from the rota-rod was automatically counted. The maximum time to fall was limited to 180 seconds.

\section{Immunohistochemistry}

Immunohistochemistry for calbindin, glial fibrillary acidic protein (GFAP), and Iba-1-positive cells in the cerebellar vermis was conducted, as the method described before $[8,20]$. The sections were treated with $0.05 \mathrm{M}$ phosphate-buffered saline (PBS) during 5 minutes, and washed three times by $0.05 \mathrm{M}$ PBS. Freefloating sections were treated with $3 \% \mathrm{H}_{2} \mathrm{O}_{2}$ during 30 minutes, and then the sections were treated with blocking solution (1\% bovine serum albumin and 10\% horse serum in $0.05 \mathrm{M}$ PBS) during 2 hours under the laboratory temperature. After treating with mouse anti-calbinind-D-28K antibody (1:500; Sigma Chemical Co.), goat anti-GFAP antibody (1:500; Chemicon International Inc., Temecula, CA, USA), and rabbit anti-Iba-1 antibody (1:500; Abcam, Cambridge, Cambridgeshire, UK) during overnight, the biotinylated anti-mouse, anti-goat, and antirabbit IgG secondary antibodies (1:200; Vector Laboratories, Burlingame, CA, USA) were treated. Avidin-biotin-peroxidase complex (Vector Laboratories) was treated to the section during 1 hour under the laboratory temperature. Following treatment with $0.02 \%$ diaminobenzidine and $0.03 \% \mathrm{H}_{2} \mathrm{O}_{2}$ in $50 \mathrm{mM}$ Tris- $\mathrm{HCl}(\mathrm{pH}, 7.6)$ during 5 minutes, the sections were treated with PBS, and then mounted onto gelatin-coated slide. Permount (Fisher Scientific, Fair Lawn, NJ, USA) was used for the mounting of coverslips.

By the Image-Pro Plus software (Media Cybernetics Inc., Silver Spring, MD, USA), we quantified the number of calbindinpositive Purkinje cells in the $1,000-\times 400-\mu \mathrm{m}$ field of the cerebellar vermis. By the quantitative fashion based on optical density, the density of GFAP-positive reactive astrocytes and Iba1-positive microglia was assessed.

\section{TUNEL Staining}

By an In Situ Cell Death Detection Kit (Roche, Mannheim, Germany), TUNEL staining was processed, as the method described before [20,21]. After treating with ethanol-acetic acid (2:1), the sections were treated with proteinase $\mathrm{K}(100 \mu \mathrm{g} / \mathrm{mL})$, treated with $3 \% \mathrm{H}_{2} \mathrm{O}_{2}$, permeabilized with $0.5 \%$ Triton X-100, and then treated with the TUNEL reaction mixture. The sections were visualized by the label fluorescein-dUTP to the 39end of DNA fragments. The fluorescent mounting medium (DakoCytomation, Carpinteria, CA, USA) was used for the mounting of coverslips. By the Image-Pro Plus software (Media Cybernetics Inc.), the number of TUNEL-positive cells in a field of $100 \mu \mathrm{m} \times 100 \mu \mathrm{m}$ of the cerebellar vermis was counted.

\section{Western Blot Analysis}

For the detection of Bax and Bcl-2 expression, we used Western blotting, as the method described before [20,21]. After separating $40-\mu \mathrm{g}$ protein on sodium dodecyl sulfate-polyacrylamide gels, protein was transferred onto a nitrocellulose membrane (Schleicher \& Schuell GmbH, Dassel, Germany). For the primary antibodies, we used mouse anti-actin antibody (1:2,000; Santa Cruz Biotechnology, Santa Cruz, CA, USA), mouse anti-Bax antibody (1:1,000; Santa Cruz Biotechnology), and mouse antiBcl-2 antibody (1:1,000 Santa Cruz Biotechnology). As the secondary antibodies, we used mouse horseradish peroxidase-conjugated antibody (1:2,000; Santa Cruz Biotechnology) for actin, $\mathrm{Bax}$, and Bcl-2. We used enhanced chemiluminescence detection system (Amersham Pharmacia Biotechnology GmbH, Freiburg, Germany) for the band detection. By Image-Pro Plus software (Media Cybernetics Inc.), the detected bands were densitometrically analyzed.

\section{Results Analysis}

For the statistical analysis, 1-way analysis of variance with the Duncan post hoc test was performed. IBM SPSS Statistics ver. 22.0 (IBM Co., Armonk, NY, USA) was used for statistical analysis. Mean \pm standard error of the mean were presented for the expression of results, and $\mathrm{P}<0.05$ was determined as a statistical significance.

\section{RESULTS}

\section{Latency of Rota-Rod Test}

Fig. 1 shows the latency of the rota-rod test. Our results showed that rotenone injection shortened the latency to fall $(\mathrm{P}<0.05)$, 


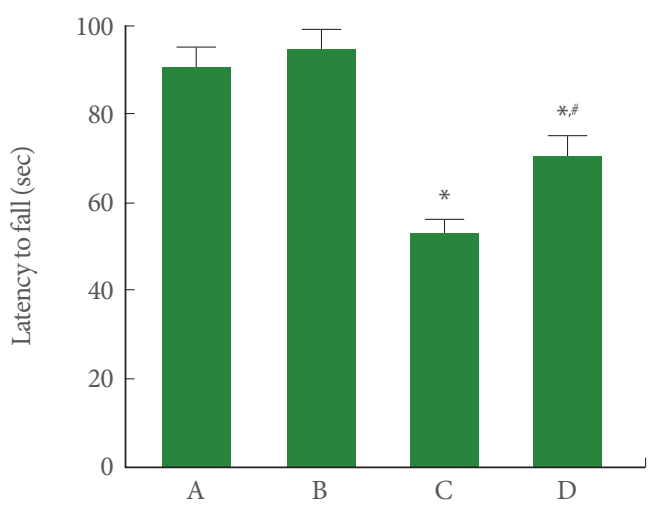

Fig. 1. Treadmill exercise alleviates motor dysfunction after rotenone in the rota-rod test. A, control group; B, treadmill exercise group; $\mathrm{C}$, rotenone-injection group; $\mathrm{D}$, rotenone-injection and treadmill exercise group. ${ }^{*} \mathrm{P}<0.05$ compared with the control group. ${ }^{*} \mathrm{P}<0.05$ compared with the rotenone-injection group.

meanwhile, treadmill running lengthened the latency to fall of the rotenone-induced $\mathrm{PD}$ rats $(\mathrm{P}<0.05)$.

\section{Purkinje Cells in Cerebellar Vermis}

Fig. 2 is photomicrographs representing calbindin-positive Purkinje cells in the cerebellar vermis. Our results showed that rotenone injection suppressed the number of Purkinje cells in the cerebellar vermis $(\mathrm{P}<0.05)$, meanwhile, treadmill running increased this Purkinje cell number in the rotenone-induced $\mathrm{PD}$ rats $(\mathrm{P}<0.05)$.

\section{Reactive Astrocytes in Cerebellar Vermis}

Fig. 3 is photomicrographs representing GFAP-positive reactive astrocytes in the cerebellar vermis. Our results showed that rotenone injection increased reactive astrocyte expression in the molecular and granular layers of cerebellar vermis $(\mathrm{P}<0.05)$, meanwhile, treadmill running inhibited this reactive astrocyte expression in the rotenone-induced $\mathrm{PD}$ rats $(\mathrm{P}<0.05)$.

\section{Microglia in Cerebellar Vermis}

Fig. 4 is photomicrographs representing Iba-1-positive microglia in the cerebellar vermis. Our results showed that rotenone injection increased the expression of microglia in the molecular and granular layers of cerebellar vermis $(\mathrm{P}<0.05)$, meanwhile, treadmill running inhibited this microglia expression in the rotenone-induced $\mathrm{PD}$ rats $(\mathrm{P}<0.05)$.

\section{TUNEL-Positive Cells in Cerebellar Vermis}

Fig. 5 is photomicrographs representing TUNEL-positive cells
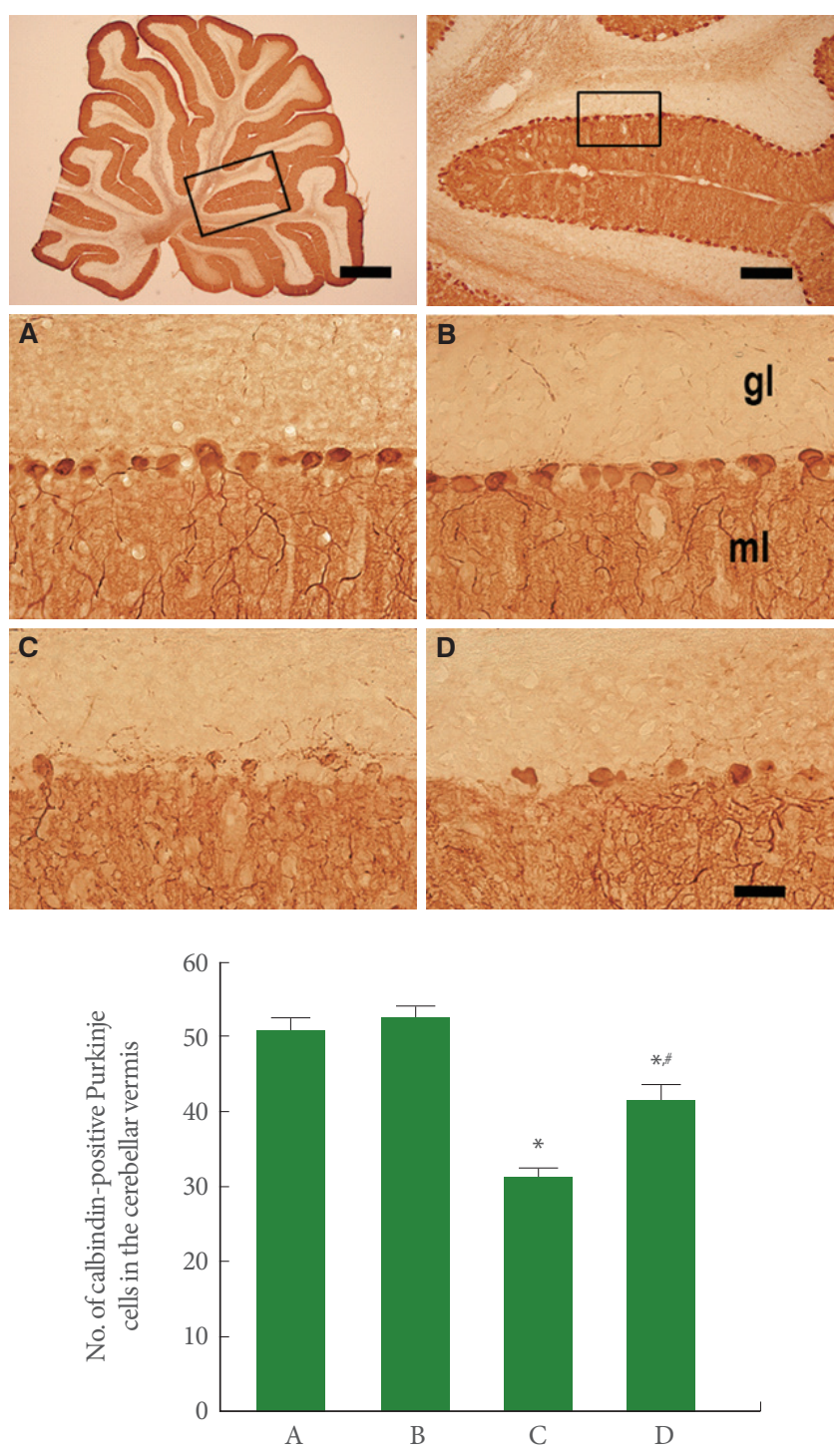

Fig. 2. Effect of treadmill exercise on Purkinje cells in the cerebellar vermis. Upper panel: Photomicrographs of calbindin immunostaining. The scale bar represents $1,000 \mu \mathrm{m}$ (upper left), $200 \mu \mathrm{m}$ (upper right), and $40 \mu \mathrm{m}$ (lower). Lower panel: The number of calbindin-positive Purkinje cells in each group. A, control group; B, treadmill exercise group; C, rotenone-injection group; $\mathrm{D}$, rotenone-injection and treadmill exercise group. ${ }^{*} \mathrm{P}<0.05$ compared with the control group. ${ }^{*} \mathrm{P}<0.05$ compared with the rotenone-injection group.

in the cerebellar vermis. Our results showed that rotenone injection enhanced the number of TUNEL-positive cells in the cerebellar vermis $(\mathrm{P}<0.05)$, meanwhile, treadmill running suppressed this TUNEL-positive cell number in the rotenone-induced $\mathrm{PD}$ rats $(\mathrm{P}<0.05)$. 

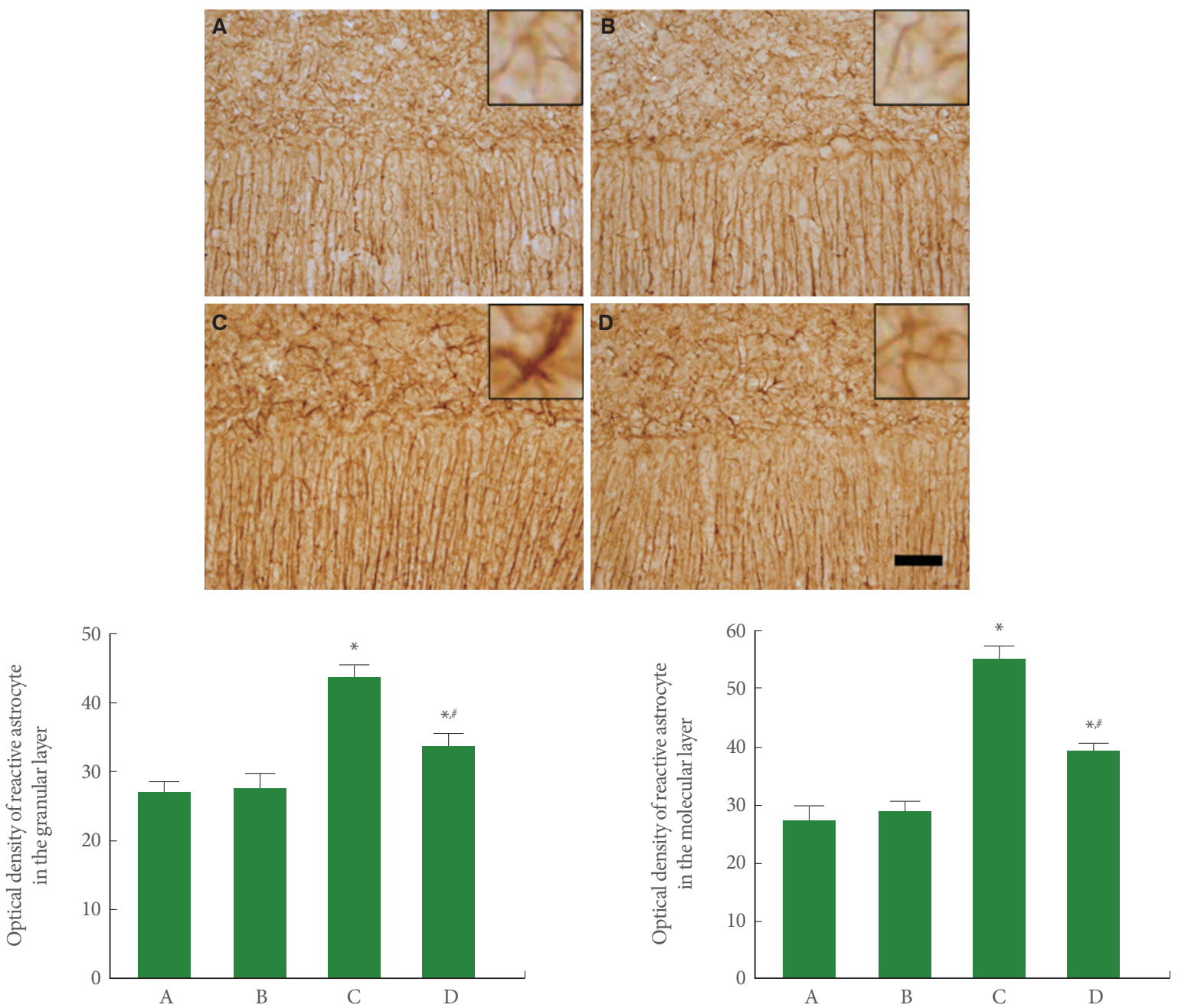

Fig. 3. Effect of treadmill exercise on reactive astrocyte in the cerebellar vermis. Upper panel: Photomicrographs of glial fibrillary acidic protein (GFAP) immunostaining. The scale bar represents $40 \mu \mathrm{m}$. Lower panel: The optical density of GFAP-positive cells in each group. A, control group; $\mathrm{B}$, treadmill exercise group; $\mathrm{C}$, rotenone-injection group; $\mathrm{D}$, rotenone-injection and treadmill exercise group. ${ }^{*} \mathrm{P}<0.05$ compared with the control group. ${ }^{*} \mathrm{P}<0.05$ compared with the rotenone-injection group.

\section{Bax and Bcl-2 Expressions in Cerebellum}

Fig. 6 is the relative expression representing Bax and Bcl-2 in the cerebellum. Our results showed that rotenone injection increased the expression of $\operatorname{Bax}(\mathrm{P}<0.05)$ and suppressed the expression of Bcl-2 in the cerebellum $(\mathrm{P}<0.05)$, meanwhile, treadmill running inhibited this Bax expression $(\mathrm{P}<0.05)$ and increased this $\mathrm{Bcl}-2$ expression $(\mathrm{P}<0.05)$ in the rotenone-induced $\mathrm{PD}$ rats $(\mathrm{P}<0.05)$.

\section{DISCUSSION}

Rotenone-induced PD rats showed decreased locomotor activity and delayed movement initiation time [4]. Rigidity, tremor, gait disturbance with non-motor symptom are caused by path- ological changes of the cerebellum in PD [2]. Parameshwaran et al. [25] suggested that rotenone injection reduced the latency to fall that was determined by rota-rod test. Rotenone-induced PD rats showed disturbed motor balance and coordination in the rota-rod test, and loss of dopaminergic neurons and fibers in the nigrostriatum was observed [23]. Treadmill running improved motor balance and coordination by suppression of Purkinje cell loss in the cerebellar vermis of the autistic rats made by valproic acid [8]. Treadmill running preserved dopaminergic neurons and fibers and inhibited formation of Lewy bodies in the nigrostriatum, and then improved motor ability in the rotenone-induced $\mathrm{PD}$ rats [23].

In our study, rotenone injection suppressed Purkinje cell 

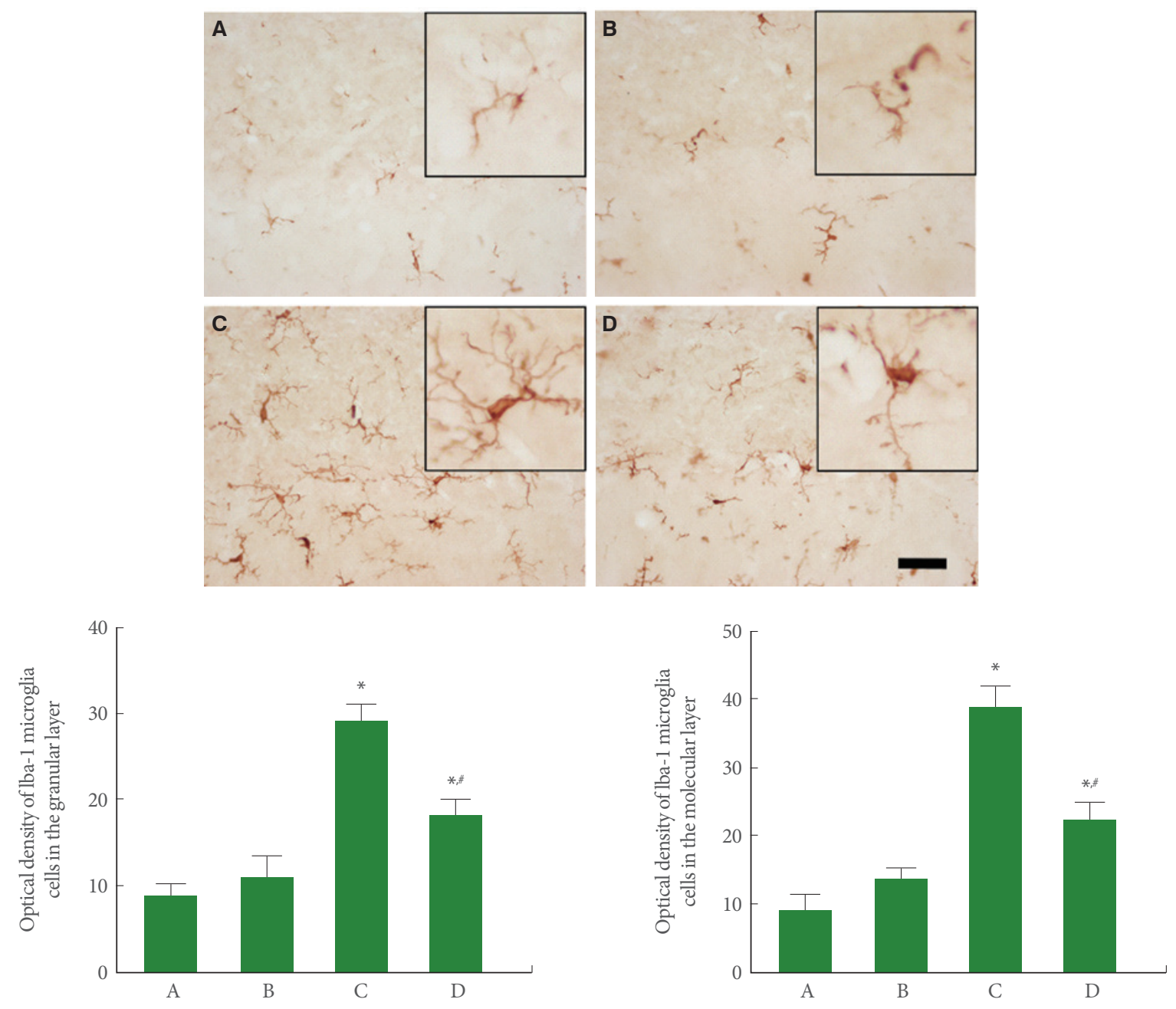

Fig. 4. Effect of treadmill exercise on microglia in the cerebellar vermis. Upper panel: Photomicrographs of Iba-1 immunostaining. The scale bar represents $40 \mu \mathrm{m}$. Lower panel: The optical density of Iba-1-positive cells in each group. A, control group; B, treadmill exercise group; C, rotenone-injection group; $\mathrm{D}$, rotenone-injection and treadmill exercise group. ${ }^{*} \mathrm{P}<0.05$ compared with the control group. ${ }^{\#} \mathrm{P}<0.05$ compared with the rotenone-injection group.

number in the cerebellar vermis. Rotenone injection also lessened the latency of the rota-rod test, suggested that rotenone impaired motor balance and coordination. In contrast, treadmill running improved this motor ability by inhibiting Purkinje cell loss in the cerebellar vermis.

Reactive astrocytes or functional activation of astrocytes was abundantly appeared in traumatic, ischemic, and Parkinson's animal models [11]. Loss of Purkinje cells in the cerebellar vermis has close relation with the reactive astrogliosis. Reactive astrogliosis is a response of astrocytes to brain injury, and GFAP is a marker of reactive astrocytes $[8,20]$. In the rotenone-induced PD rats, excessive microglial activation appeared in the striatum and substantia nigra [16]. Immunorectivity to Iba1 protein represents microglia, and Ibal regulates the function of activated microglia [13]. Microglial activation is the main pathologic event for loss of dopaminergic neurons in the PD mice [17]. The memory enhancing effect of treadmill running may be due to the exercise-mediated inhibition of reactive astrocytes and microglia $[8,17]$.

In our study, the expression of GFAP-positive reactive astrocytes and Iba-1-positive microglia in the cerebellar vermis of rotenone-induced PD rats was increased, demonstrating that rotenone injection induced reactive astrogliosis and microglia activation. In contrast, treadmill running suppressed PD-induced expression of GFAP-positive reactive astrocytes and Iba1-positive microglia, showing that treadmill exercise suppressed 

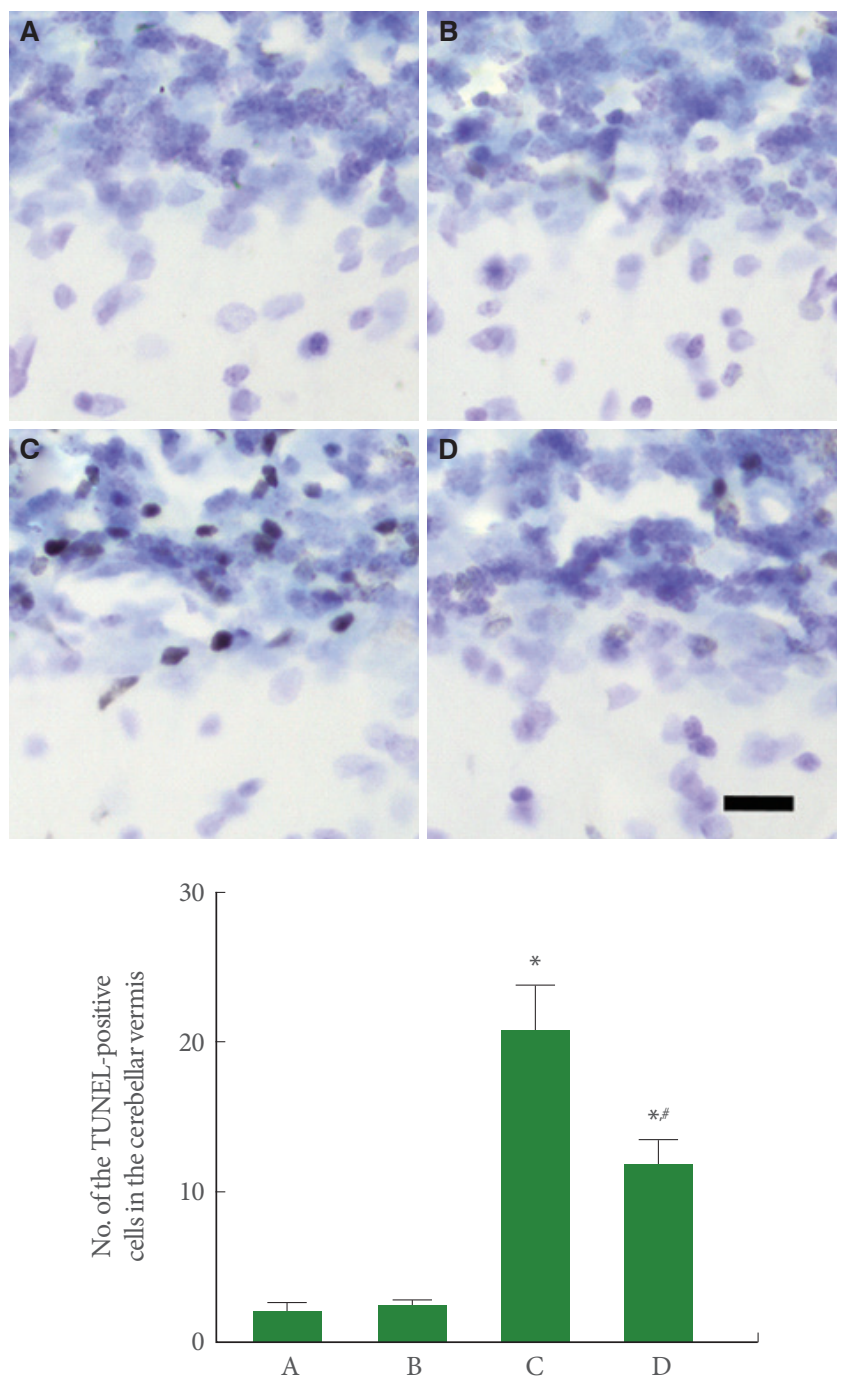

Fig. 5. Effect of treadmill exercise on terminal deoxynucleotidyl transferase-mediated dUTP nick end labeling (TUNEL)-positive cells in the cerebellar vermis. Upper panel: Photomicrographs of TUNEL immunostaining. The scale bar represents 10 $\mu \mathrm{m}$. Upper panel: The number of TUNEL-positive cells in each group. A, control group; $\mathrm{B}$, treadmill exercise group; $\mathrm{C}$, rotenone-injection group; $\mathrm{D}$, rotenone-injection and treadmill exercise group. ${ }^{*} \mathrm{P}<0.05$ compared with the control group. ${ }^{*} \mathrm{P}<0.05$ compared with the rotenone-injection group.

reactive astrogliosis and microglia activation.

TUNEL-positive cells indicate DNA fragmentation, which represents apoptosis, and then decrease of TUNEL-positive cell number indicates inhibition of apoptosis [20,21]. Increase of pro-apoptotic Bax expression promotes apoptosis, in contrast, increase of antiapoptotic Bcl-2 expression inhibits apoptosis [20,21]. Apoptosis pathways in the Parkinson's disease include signaling by $\mathrm{Bcl}-2$
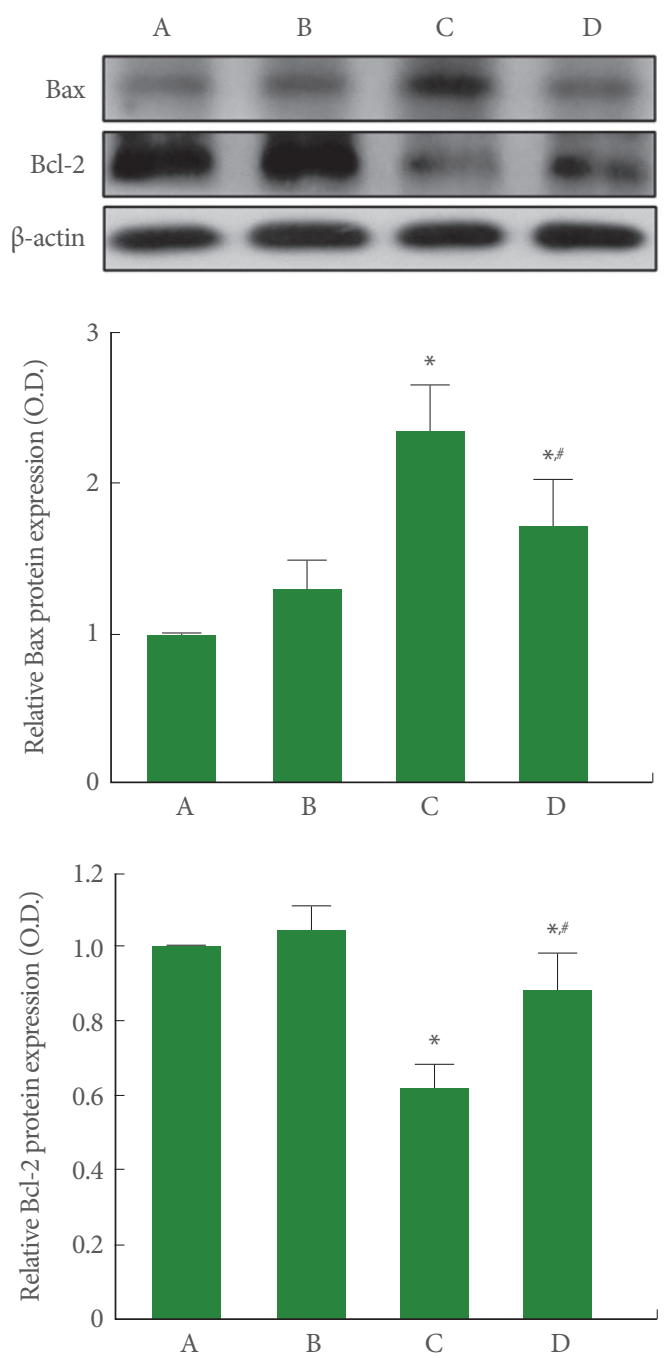

Fig. 6. Effect of treadmill exercise on the expressions of Bax and Bcl-2 in the cerebellum. Upper panel: Representative Bax and Bcl-2 expressions. Middle panel: Relative optical density of Bax in each group. Lower panel: Relative optical density of Bcl-2 in each group. A, control group; $\mathrm{B}$, treadmill exercise group; $\mathrm{C}$, rotenone-injection group; $\mathrm{D}$, rotenone-injection and treadmill exercise group. ${ }^{*} \mathrm{P}<0.05$ compared with the control group. ${ }^{\#} \mathrm{P}<0.05$ compared with the rotenone-injection group.

family proteins [19]. Bax up-regulation and Bcl-2 down-regulation in the SK-N-SH cells were appeared in the rotenone-induced apoptosis [26]. Enhanced Bax expression and suppressed Bcl-2 expression were occurred in the brain of PD animals [18,27]. Treadmill running inhibited Bax expression and increased $\mathrm{Bcl}-2$ expression in the cerebellum of depressive rats [21].

In our study, TUNEL-positive cell number and Bax expres- 
sion were enhanced and Bcl-2 expression was inhibited in the cerebellum of rotenone-induced PD rats. Our present results reveled that rotenone injection facilitated the progress of apoptosis in the cerebellum. In contrast, treadmill running suppressed these TUNEL-positive cell number and Bax expression and enhanced Bcl-2 expression, demonstrating that treadmill running inhibited the progress of apoptosis in the cerebellum of rotenone-induced $\mathrm{PD}$ rats.

Presently, treadmill running improved motor ability in the rotenone-induced PD rats by inhibiting apoptosis in the cerebellum. Apoptosis suppressing effect of treadmill running on rotenone-induced PD was achieved via suppression of reactive astrocyte and inhibition of microglial activation. According to our results, exercise can be considered as a treatment modality for the patients with $\mathrm{PD}$.

\section{AUTHOR CONTRIBUTION STATEMENT}

- Full access to all the data in the study and takes responsibility for the integrity of the data and the accuracy of the data analysis: JML, KHK

- Study concept and design: JML

- Acquisition of data: TWK, SSP, SHK

- Analysis and interpretation of data: JHH, MSS, YSC

- Drafting of the manuscript: $J M L$

- Critical revision of the manuscript for important intellectual content: $S H K, S S B$

- Statistical analysis: $B V L$

- Obtained funding: $\mathrm{KHK}$

- Administrative, technical, or material support: JHH, BVL, SSB, YSC

- Study supervision: $K H K$

\section{REFERENCES}

1. Olanow CW, Stern MB, Sethi K. The scientific and clinical basis for the treatment of Parkinson disease (2009). Neurology 2009;72(21 Suppl 4):S1-136.

2. Wu T, Hallett M. The cerebellum in Parkinson's disease. Brain 2013;136(Pt 3):696-709.

3. Dauer W, Przedborski S. Parkinson's disease: mechanisms and models. Neuron 2003;39:889-909.

4. Fleming SM, Zhu C, Fernagut PO, Mehta A, DiCarlo CD, Seaman $\mathrm{RL}$, et al. Behavioral and immunohistochemical effects of chronic intravenous and subcutaneous infusions of varying doses of rote- none. Exp Neurol 2004;187:418-29.

5. Barski JJ, Hartmann J, Rose CR, Hoebeek F, Mörl K, Noll-Hussong $\mathrm{M}$, et al. Calbindin in cerebellar Purkinje cells is a critical determinant of the precision of motor coordination. J Neurosci 2003;23: 3469-77.

6. Heman P, Barcia C, Gómez A, Ros CM, Ros-Bernal F, Yuste JE, et al. Nigral degeneration correlates with persistent activation of cerebellar Purkinje cells in MPTP-treated monkeys. Histol Histopathol 2012;27:89-94.

7. Yan J, Fu Q, Cheng L, Zhai M, Wu W, Huang L, Du G. Inflammatory response in Parkinson's disease (Review). Mol Med Rep 2014; 10:2223-33.

8. Cho HS, Kim TW, Ji ES, Park HS, Shin MS, Baek SS. Treadmill exercise ameliorates motor dysfunction through inhibition of Purkinje cell loss in cerebellum of valproic acid-induced autistic rats. J Exerc Rehabil 2016;12:293-8.

9. Maragakis NJ, Rothstein JD. Mechanisms of disease: astrocytes in neurodegenerative disease. Nat Clin Pract Neurol 2006;2:679-89.

10. Barreto GE, White RE, Xu L, Palm CJ, Giffard RG. Effects of heat shock protein 72 (Hsp72) on evolution of astrocyte activation following stroke in the mouse. Exp Neurol 2012;238:284-96.

11. Chen LW, Yung KL, Chan YS. Reactive astrocytes as potential manipulation targets in novel cell replacement therapy of Parkinson's disease. Curr Drug Targets 2005;6:821-33.

12. Sun XL, Chen BY, Zhao HK, Cheng YY, Zheng MH, Duan L, et al. Gas1 up-regulation is inducible and contributes to cell apoptosis in reactive astrocytes in the substantia nigra of LPS and MPTP models. J Neuroinflammation 2016;13:180.

13. Ito D, Imai Y, Ohsawa K, Nakajima K, Fukuuchi Y, Kohsaka S. Microglia-specific localisation of a novel calcium binding protein, Iba1. Brain Res Mol Brain Res 1998;57:1-9.

14. Ohsawa K, Imai Y, Kanazawa H, Sasaki Y, Kohsaka S. Involvement of Ibal in membrane ruffling and phagocytosis of macrophages/ microglia. J Cell Sci 2000;113(Pt 17):3073-84.

15. Gao HM, Hong JS, Zhang W, Liu B. Distinct role for microglia in rotenone-induced degeneration of dopaminergic neurons. J Neurosci 2002;22:782-90.

16. Sherer TB, Betarbet R, Kim JH, Greenamyre JT. Selective microglial activation in the rat rotenone model of Parkinson's disease. Neurosci Lett 2003;341:87-90.

17. Sung YH, Kim SC, Hong HP, Park CY, Shin MS, Kim CJ, et al. Treadmill exercise ameliorates dopaminergic neuronal loss through suppressing microglial activation in Parkinson's disease mice. Life Sci 2012;911309-16.

18. Kim M, Cho KH, Shin MS, Lee JM, Cho HS, Kim CJ, et al. Berber- 
ine prevents nigrostriatal dopaminergic neuronal loss and suppresses hippocampal apoptosis in mice with Parkinson's disease. Int J Mol Med 2014;33:870-8.

19. Levy OA, Malagelada C, Greene LA. Cell death pathways in Parkinson's disease: proximal triggers, distal effectors, and final steps. Apoptosis 2009;14:478-500.

20. Baek SS, Kim SH. Treadmill exercise ameliorates symptoms of Alzheimer disease through suppressing microglial activation-induced apoptosis in rats. J Exerc Rehabil 2016;12:526-34.

21. Roh JH, Ko IG, Kim SE, Lee JM, Ji ES, Kim JH, et al. Treadmill exercise ameliorates intracerebral hemorrhage-induced depression in rats. J Exerc Rehabil 2016;12:299-307.

22. Shin MS, Ko IG, Kim SE, Kim BK, Kim TS, Lee SH, et al. Treadmill exercise ameliorates symptoms of methimazole-induced hypothyroidism through enhancing neurogenesis and suppressing apoptosis in the hippocampus of rat pups. Int $J$ Dev Neurosci 2013;31:21423.

23. Shin MS, Kim TW, Lee JM, Ji ES, Lim BV. Treadmill exercise allevi- ates nigrostriatal dopaminergic loss of neurons and fibers in rotenone-induced Parkinson rats. J Exerc Rehabil 2017;13:30-5.

24. Jung SY, Kim DY. Treadmill exercise improves motor and memory functions in cerebral palsy rats through activation of PI3K-Akt pathway. J Exerc Rehabil 2017;13:136-42.

25. Parameshwaran K, Irwin MH, Steliou K, Pinkert CA. Protection by an antioxidant of rotenone-induced neuromotor decline, reactive oxygen species generation and cellular stress in mouse brain. Pharmacol Biochem Behav 2012;101:487-92.

26. Tamilselvam K, Braidy N, Manivasagam T, Mohamed M. Neuroprotective effects of hesperidin, a plant flavanone on rotenone induced oxidative stress and apoptosis in a cellular model for Parkinson's disease. Oxid Med Cell Longev 2013;2013:102741.

27. Xu CL, Wang QZ, Sun LM, Li XM, Deng JM, Li LF, et al. Asiaticoside: Attenuation of neurotoxicity induced by MPTP in a rat model of Parkinsonism via maintaining redox balance and up-regulating the ratio of Bcl-2/Bax. Pharmacol Biochem Behav 2012;100:413-8. 